\title{
Culturable fungal diversity associated with forest leaf litter from Bhandara District of Maharashtra, India
}

\author{
SUNIL M. AKARE ${ }^{1}$, WALAY Y. TAGADE ${ }^{2}$, ASHISH R. WARGHAT ${ }^{3, \bullet}$, AVILEKH NARYAL $^{3}$, \\ ASHWANI BHARDWAJ ${ }^{3}$ \\ ${ }^{1}$ Department of Botany, M. B. Patel College, Sadak Arjuni-441807, Maharashtra, India \\ ${ }^{2}$ Department of Botany, C. J. Patel College, Tirora-441911, Maharashtra, India \\ ${ }^{3}$ Defence Institute of High Altitude Research, Defence R \& D Organization, Leh-Ladakh-194101, Jammu \& Kashmir, India. Tel./Fax.: +91-1982- \\ 252096, ”email: ashishwarghat@hotmail.com
}

Manuscript received: 14 October 2015. Revision accepted: 17 April 2016.

\begin{abstract}
Akare SM, Tagade WY, Warghat AR, Naryal A, Bhardwaj A. 2016. Culturable fungal diversity associated with forest leaf litter from Bhandara District of Maharashtra, India. Biodiversitas 17: 349-358. Diversity of leaf litter fungi were carried out in 201011. Four samples were collected from Bampewada and Sakoli forest area of Bhandara District of Maharashtra, India. The Blotter paper method, Segment plate method and Serial dilution methods were used to assess the diversity of fungal species. Fungi were cultivated on potato dextrose agar, malt extract agar and czapek dox agar. A total of 31 species comprising 29 Ascomycetes and two Zygomycetes were recognized from 4 samples collected from Bampewada and Sakoli forest area. The most abundant group was Ascomycetes. The dominant species in both the forests were A. flavus, A. niger, Aureobasidium sp., Fusarium oxysporum, Penicillium sp.1, Rhizopus stolonifer, A. caespitosus, Penicillium sp.2, A. nidulans, Helminthosporium sp. and Monodictys fluctuata. While, common species were A. flavus, A. fumigatus, A. nidulans, A. niger, Beltrania rhombica and Fusarium oxysporum. The fungal diversity was higher in the Sakoli forest than that of Bampewada forest. Differences were observed in percentage occurrence of fungal species between two forest areas. The reason may be that the quality of litters, different microenvironments and other characteristics in the Sakoli forest provided more resources for fungi than in Bampewada forest.
\end{abstract}

Keywords: Decomposition, dominant species, fungal community, litter breakdown, vegetation

\section{INTRODUCTION}

Fungi play an important role in balancing ecological services, their utilization in industry, agriculture, medicine, food industry, textiles, bioremediation, natural cycling and decomposing the dead organic matter present in soil and litter (Change and Miles 2004; Gates 2005). They are highly diverse in nature. Having a stable and estimate of taxonomic diversity for fungi is also necessary to enable fungi to be included in considerations of biodiversity conservation, land-use planning and management (Mueller and Schmit 2007).

The number of existing fungi worldwide has been estimated to 1.5 million species (Mueller et al. 2004). Onethirds of the fungal diversity of the globe exists in India and of this, only $50 \%$ are characterized yet (Manoharachary et al. 2005). Maharashtra is the third largest state of India next to Rajasthan and Madhya Pradesh covering an area of $307,713 \mathrm{~km}^{2}$. It lies at $18^{\circ} 5736 \mathrm{~N} 72^{\circ} 4912 \mathrm{E}$, and altitude ranges from $0-1800 \mathrm{~m}$ above mean sea level and the forests cover less than one fifth of the state and confined to the Western Ghats and eastern Vidarbha region. It receives an annual rain fall of about $4000 \mathrm{~mm}$ in the western region of Western Ghats and about 700-1250 $\mathrm{mm}$ in Vidarbha region brought by south west monsoon and the dry zone occurs in between western and Vidarbha region. The forests of Western Ghats and
Vidarbha region are rich in mycobiota (Senthilarasu 2014).

Decomposition of fungi on the forest floor is a very complex phenomenon and achieved by different groups of microorganisms. The major component of the top soil consists of different parts of plant materials and immediately colonized by diverse groups of microorganisms as they fall on the soil surface and soon after, the processes of decomposition starts. Litter decomposition is also an important link in nutrient cycling of the forest (Grigal and McColl 1977). Litter is an important source of dead organic matter in terrestrial ecosystems, with inputs of tons of litter per year. Litter decomposition contributes directly to nutrient availability both for plant growth and ecosystem productivity. The studies on microorganisms suggest fungi to be the main contributors to leaf litter decomposition (Isidorov and Jdanova 2002). During the last few years various workers have developed interest to understand the nature of fungi both in forest and cultivated fields. The study on diversity of leaf litter fungi from various host plants were reported earlier (Saravanan 2013; Tokumasu et al. 1997). Some fungi were found to be common on leaf litter in previous studies, while many new fungal taxa have been described from decaying leaves and dead wood (Hughes 1989). Keeping the above facts in mind, the present study was focused on the isolation and identification of fungi associated with decomposition of forest leaf litter in 
Bampewada and Sakoli area of Bhandara District of Maharashtra, India. Forest leaf-litter was selected for the present study because of the dense forest vegetation and its great economic value in Maharashtra, India.

\section{MATERIALS AND METHODS}

\section{Study area}

Present investigation embodies isolation and identification of fungi associated with forest leaf litter. The samples were collected from forest of Bampewada and Sakoli area of Bhandara District, Maharashtra State, India (Figure 1). For the collection of leaf litter samples, two places were selected namely, Bampewada and Sakoli forest (Figure 2). These two places are $53 \mathrm{~km}$ and $40 \mathrm{~km}$ away from Bhandara District of Maharashtra State. The forest areas are entirely dominated by Teak trees however; other tree species such as Babul, Ber, Palas, Shisham, Amla, Jamun, Arjun, Ain, Chichwa, Sewan, Dhaora, Tendu, Salai, Rohan, Mahua, Bija, Dhaman, etc. are also found. The climate in this area remains dry and hot throughout the year with the moderate rainfall from June to middle of October months. In each places two locations namely hilly region and open slopes were selected randomly for collections of leaf litter which were appropriate from ecological point of view (dry, hot and moderate rainfall during the monsoon). Collections were made before annual leaf fall starts particularly, in the first week of January. At the time of collections, partial decomposed leaves (for chances of presence of mycelium) were collected in a sterile plastic ziploc bags (ziplocs bags were sterilized by absolute alcohol) of size $13 \mathrm{~cm} \times 10 \mathrm{~cm}$ and brought to the laboratory for fungal isolation.

\section{Culture media}

The following culture media were used for the isolation of fungi

\section{Potato Dextrose Agar (Rawling 1933)}

This culture medium is used for the isolation of fungi from forest leaf litter by serial dilution method. The compositions of Potato Dextrose Agar are as follows: Potatoes (200g, Dextrose (20g), Agar 17g (Distilled water (1000 mL).

\section{Malt Extract Agar (Van der Walt \& Yarrow 1984)}

This culture medium is used for isolation of fungi by segment plate method. The compositions of Malt Extract Agar are as follows: Malt Extract (20 g), Agar (17 g), Distilled water $(1000 \mathrm{~mL})$.

\section{Czapek Dox Agar (Raper \& Thom 1949)}

This culture medium is used for the isolation of fungi by segment plate method and pure culture of fungal colonies. The compositions of Czapek Dox Agar are as follows: $\mathrm{NaNO}_{3}(3.00 \mathrm{~g}), \mathrm{K}_{2} \mathrm{HPO}_{4}(1.00 \mathrm{~g}), \mathrm{MgSO}_{4} .7 \mathrm{H}_{2} \mathrm{O}$ $(0.5 \mathrm{~g}), \mathrm{KCl}(0.5 \mathrm{~g}), \mathrm{FeSO}_{4} .7 \mathrm{H}_{2} \mathrm{O}(0.01 \mathrm{~g})$, Sucrose (30.00 g), Agar (15.00 g), Distilled water (1000 mL).

\section{Methods for isolation \\ Moist chamber technique (Blotter paper method) (Fulzele 2002)}

The collected samples were aseptically transferred to the sterile petriplate containing wet blotter papers and were kept at room temperature in the incubation chamber. Sterile distilled water was added at regular interval under aseptic condition to maintain blotter paper moist.

\section{Direct plating method (Segment plate method) (Hutchinson and Richard 1921)}

Randomly 4-5 segments of material nearly of size $1 / 2$ to 1 inch were directly placed on culture petriplate containing sterile, cool, solid Malt Extract Agar medium at equidistance from one another and incubated at room temperature. The care was taken to avoid overcrowding of fungi in the plate.

\section{Serial dilution methods (Hutchinson and Richard 1921)}

$1 \mathrm{gm}$ of material from collected samples was mixed with $10 \mathrm{~mL}$ sterile distilled water with continuous stirring for obtaining homogenous suspension of fungal spores. $1 \mathrm{~mL}$ of this homogenate was transferred to first dilution tube containing $9 \mathrm{~mL}$ sterile distilled water. The tube was shaken well to get homogenate and again dilution process was carried out in the same way till the dilution reached to 1: $100000\left(10^{-5}\right)$. Afterwards, $1 \mathrm{~mL}$ of homogenate from each dilution tube was added to the sterile petriplate before pouring the sterile, cool, molten (approximately $45^{\circ} \mathrm{C}$ ) PDA medium. The plates were rotated for a brief period to proper mixing of homogenate with the medium and then allowed to cool. Then the plates were kept in incubation chamber at the room temperature of $25-30^{\circ} \mathrm{C}$. The colonies of fungi were counted per plates and were subcultured on the sterile solid, CDA medium. The Czapek Dox Agar medium was used for the growth of fungi Borkar (2014) and Fulzele (2002). The culture plates were observed at the interval of two days for a week and growing individual colonies were picked up and transferred to fresh sterile agar slants for further purification and identification.

\section{Identification of fungi}

Fungi were identified on the basis of their growth characteristics, morphological characteristics and ontogeny with the help of manuals, monographs and taxonomic papers of various authors (Ainsworth et al. 1972; Barnett \& Hunter 1972; Sutton 1980; Von Arx 1981). Identification was based on morphological study examined under stereo, and compound microscopes (Olympus BX 50 F4, Japan and Axio Scope A, Carl Zeiss). Colony forming unit and occurrence of fungi were calculated as per the procedures described by Saksena (1955). Where Percentage of occurrence refers to the number of samplings in which a fungus was recorded out of the total number of samplings made during the period of study.

The Colony Forming Unit (CFU) of fungal species per gram of leaf litter were calculated as follows:

$\mathrm{CFU} / \mathrm{gm}=$ Average number of fungal colonies $\times$ Dilution Factor Dry weight of sample 


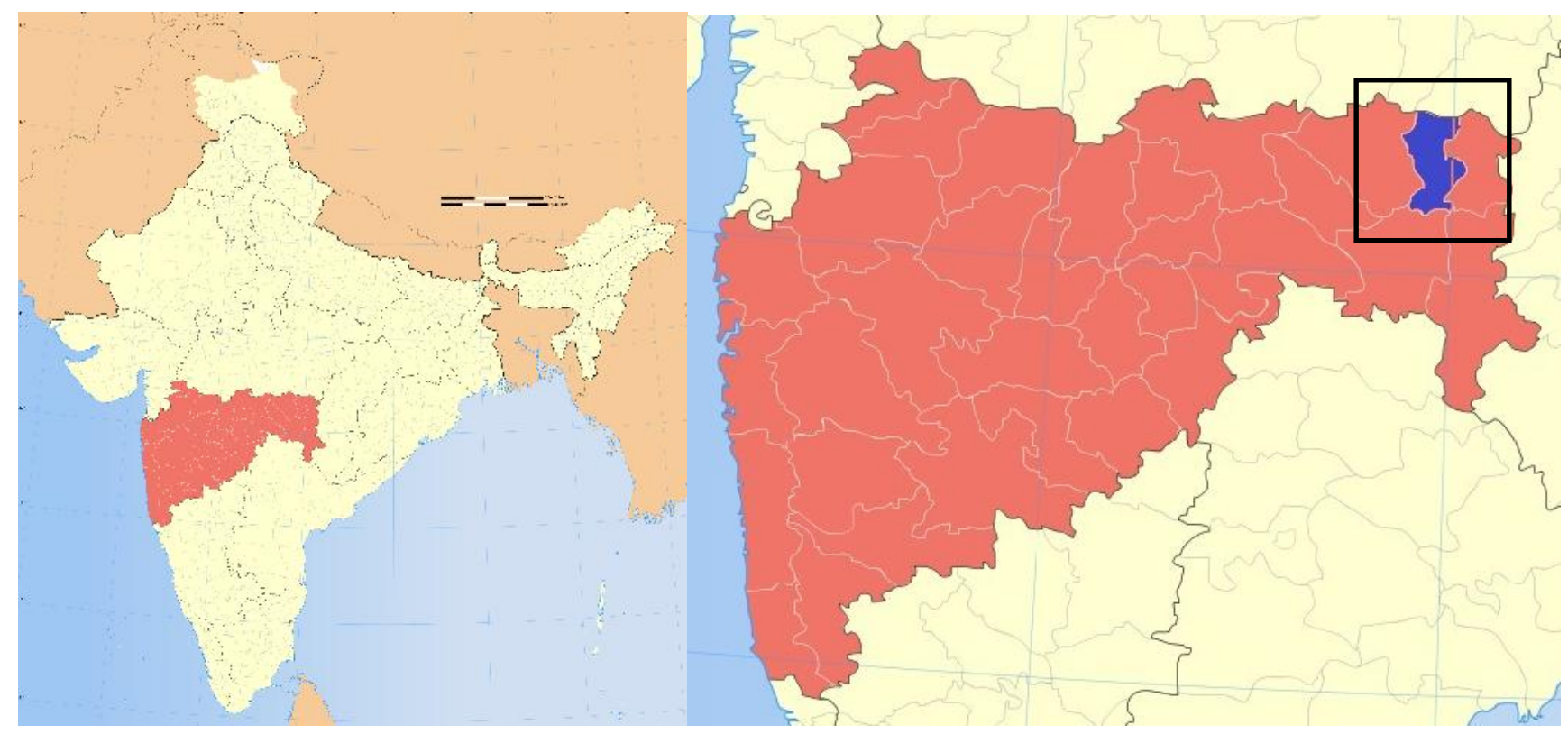

Figure 1. Location of the study area in Bampewada and Sakoli forests of Bhandara District (box), Maharashtra, India

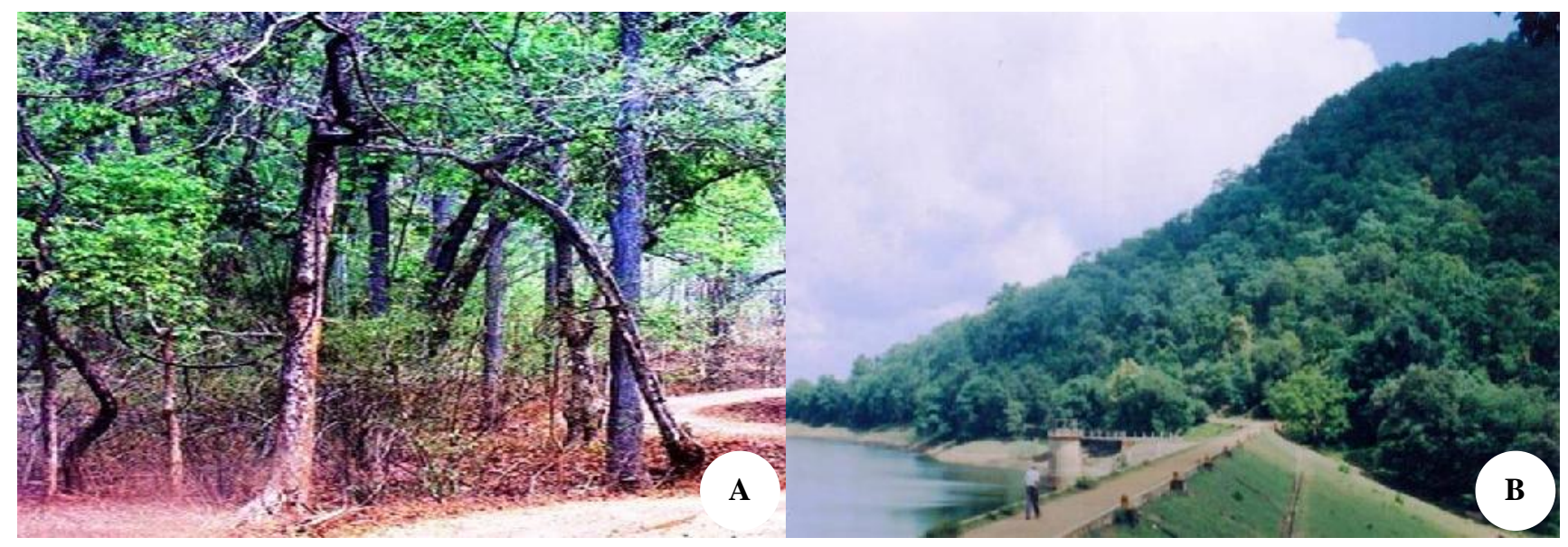

Figure 2. Studied forest area. A. Bampewada, B. Sakoli, Bhandara district of Maharashtra, India

\section{RESULTS AND DISCUSSION}

\section{Leaf litter fungi of Bampewada Forest}

Twenty six species belonging to Seventeen genera of leaf litter fungi were isolated by Serial dilution method using Potato Dextrose Agar medium (Figure 3.C). Similarly 20 species belonging to 15 genera of leaf litter fungi isolated by Segment plate method using Malt Extract Agar and Czapek Dox Agar medium (Figure 3.B). While only 4 species belonging to 4 genera of leaf litter fungi were isolated by blotter paper method (Figure 3.A). Fungi namely Alternaria sp.1, Alternaria sp.2, Aspergillus flavus, A. niger, A. ochraceus, Aureobasidium sp., Beltrania rhombica, Chaetomium sp., Curvularia sp., Fusarium oxysporum, Fusarium sp.1, Helminthosporium sp., Humicola sp., Penicillium sp.1, Penicillium sp.2, Pestalotia sp., Phoma sp., Stachybotris sp., Trichoderma sp.1,
Trichoderma sp.2 and Rhizopus stolonifer were isolated by serial dilution and segment plate method. Whereas Aspergillus caespitosus, A. fumigatus, A. nidulans, Memnoniella sp. and Syncephalastrum sp. were isolated by Serial dilution method and only Memnoniella sp., Pestalotia sp., Stachybotris sp. and Trichoderma sp.1 were isolated by Blotter paper method. While, Monodictys fluctuata, Penicillium adametzi, Pithomyces sp., Torula sp. and Trichoderma sp.3 were not identified from leaf litter fungi of Bampewada Forest by any of three method (Table 1). During the isolation of fungi it is found that A. flavus, A. niger, Aureobasidium sp., Fusarium oxysporum, Penicillium sp.1 and Rhizopus stolonifer were dominant and Aureobasidium sp.was highest colony forming fungi followed by Fusarium oxysporum, A. flavus, A. niger, Rhizopus stolonifer and Penicillium sp.1 in Bampewada forest area. Whereas, others showed least CFU (Table 2). 

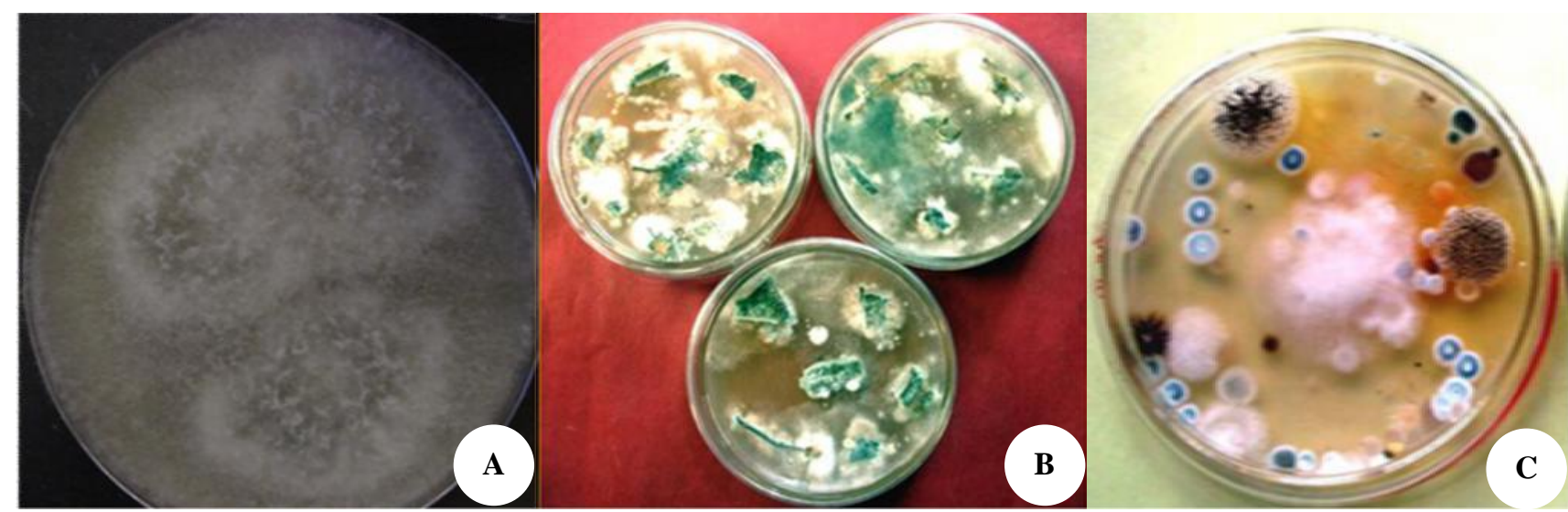

Figure 3. Isolation of fungi (samples of Bampewada and Sakoli forest area). A. Blotter paper method, B. Segment plate method, C. Serial dilution method

Table 1. Total number of fungal species isolated by different methods in selected forest area

\begin{tabular}{|c|c|c|c|c|c|c|}
\hline \multirow{2}{*}{ Fungi identified } & \multicolumn{3}{|c|}{$\begin{array}{c}\text { Bampewada } \\
\text { Forest }\end{array}$} & \multicolumn{3}{|c|}{ Sakoli forest } \\
\hline & $\mathbf{A}$ & $\mathbf{B}$ & $\mathbf{C}$ & $\mathbf{A}$ & $\mathbf{B}$ & $\mathbf{C}$ \\
\hline Alternaria sp.1 & - & + & + & - & + & + \\
\hline Alternaria sp.2 & - & + & + & - & - & - \\
\hline Aspergillus caespitosus & - & - & + & - & - & + \\
\hline Aspergillus flavus & - & + & + & + & + & + \\
\hline Aspergillus fumigatus & - & - & + & - & + & + \\
\hline Aspergillus nidulans & - & - & + & - & - & + \\
\hline Aspergillus niger & - & + & + & - & + & + \\
\hline Aspergillus ochraceus & - & + & + & - & + & + \\
\hline Aureobasidium sp. & - & + & + & - & + & + \\
\hline Beltrania rhombica & - & + & + & - & - & - \\
\hline Chaetomium sp. & - & + & + & + & - & + \\
\hline Curvularia sp. & - & + & + & - & + & + \\
\hline Fusarium oxysporum & - & + & + & - & + & + \\
\hline Fusarium sp.1 & - & + & + & - & + & + \\
\hline Helminthosporium sp. & - & + & + & - & + & + \\
\hline Humicola sp. & - & + & + & - & - & - \\
\hline Memnoniella sp. & + & - & + & + & - & + \\
\hline Monodictys fluctuata & - & - & - & - & + & + \\
\hline Penicillium adametzi & - & - & - & - & + & + \\
\hline Penicillium sp.1 & - & + & + & - & + & + \\
\hline Penicillium sp.2 & - & + & + & - & - & + \\
\hline Pestalotia sp. & + & + & + & - & + & + \\
\hline Phoma sp. & - & + & + & + & + & + \\
\hline Pithomyces sp. & - & - & - & - & + & + \\
\hline Stachybotrys sp. & + & + & + & + & - & + \\
\hline Torula sp. & - & - & - & - & - & + \\
\hline Trichoderma sp.1 & + & + & + & + & + & + \\
\hline Trichoderma sp.2 & - & + & + & - & + & + \\
\hline Trichoderma sp.3 & - & - & - & - & + & + \\
\hline Rhizopus stolonifer & - & + & + & - & + & + \\
\hline Syncephalastrum sp. & - & - & + & - & + & + \\
\hline
\end{tabular}

Table 2. Colony Forming Unit (CFU) of fungal colonies at $10^{-4}$ concentration

\begin{tabular}{|c|c|c|c|c|c|}
\hline \multicolumn{2}{|c|}{ Fungi isolates } & \multicolumn{2}{|c|}{$\begin{array}{c}\text { Bampewada } \\
\text { Forest }\end{array}$} & \multicolumn{2}{|c|}{$\begin{array}{l}\text { Sakoli } \\
\text { Forest }\end{array}$} \\
\hline Fungi identified & Division & I & II & I & II \\
\hline Alternaria sp.1 & Ascomycota & 4.17 & - & - & - \\
\hline Alternaria sp.2 & Ascomycota & - & - & - & - \\
\hline Aspergillus caespitosus & Ascomycota & - & - & 6.67 & 11.1 \\
\hline Aspergillus flavus & Ascomycota & 11.12 & 13.7 & 11.13 & 11.1 \\
\hline Aspergillus fumigatus & Ascomycota & - & 5.88 & 4.46 & 5.57 \\
\hline Aspergillus nidulans & Ascomycota & 6.95 & - & 13.23 & - \\
\hline Aspergillus niger & Ascomycota & 13.87 & 9.82 & 13.23 & 11.32 \\
\hline Aspergillus ochraceus & Ascomycota & - & - & - & 4.43 \\
\hline Aureobasidium sp. & Ascomycota & 12.5 & 13.7 & - & - \\
\hline Beltrania rhombica & Ascomycota & 3.12 & - & 3.46 & 3.23 \\
\hline Chaetomium sp. & Ascomycota & - & 5.88 & - & - \\
\hline Curvularia sp. & Ascomycota & - & - & - & 3.33 \\
\hline Fusarium oxysporum & Ascomycota & 12.5 & 13.4 & 12.33 & 13.23 \\
\hline Fusarium sp.1 & Ascomycota & 4.17 & - & 4.46 & 2.23 \\
\hline Helminthosporium sp. & Ascomycota & - & - & 6.67 & 5.57 \\
\hline Humicola sp. & Ascomycota & - & - & 2.15 & 2.33 \\
\hline Memnoniella sp. & Ascomycota & 2.5 & 3.2 & - & - \\
\hline Monodictys fluctuata & Ascomycota & - & 11.25 & 12.21 & - \\
\hline Penicillium adametzi & Ascomycota & - & - & 6.67 & - \\
\hline Penicillium sp.1 & Ascomycota & 11.12 & - & - & 10 \\
\hline Penicillium sp. 2 & Ascomycota & 8.33 & 11.76 & 13.33 & - \\
\hline Pestalotia sp. & Ascomycota & 5.54 & - & - & - \\
\hline Phoma sp. & Ascomycota & - & 3.94 & - & 4.43 \\
\hline Pithomyces sp. & Ascomycota & - & - & - & 2.23 \\
\hline Stachybotrys sp. & Ascomycota & - & 1.32 & - & 1.59 \\
\hline Torula sp. & Ascomycota & - & - & - & 4.43 \\
\hline Trichoderma sp.1 & Ascomycota & - & - & - & 2.23 \\
\hline Trichoderma sp.2 & Ascomycota & - & 3.94 & - & - \\
\hline Trichoderma sp.3 & Ascomycota & - & - & - & - \\
\hline Rhizopus stolonifer & Zygomycota & 8.33 & 3.94 & - & 5.57 \\
\hline Syncephalastrum sp. & Zygomycota & - & 5.88 & - & - \\
\hline
\end{tabular}

Note: A. Blotter paper method, B. Segment plate method, C.

Serial dilution method

\section{Leaf litter fungi of Sakoli Forest}

Twenty eight species belonging to 18 genera of leaf litter fungi were isolated by Serial dilution method using Potato Dextrose Agar medium. Whereas 21 species belonging to 14 genera of leaf litter fungi were isolated by Segment plate method using Malt Extract Agar and Czapek Dox Agar medium. While only 6 species belonging to 6 genera of fungi associated with leaf litter were isolated by 
Blotter paper method. These are Alternaria sp.1, A. flavus, A. fumigatus, A. niger, A. ochraceus, Aureobasidium sp., Curvularia sp., Fusarium oxysporum, Fusarium sp.1, Helminthosporium sp., Monodictys fluctuata, Penicillium adametzi, Penicillium sp.1, Pestalotia sp., Phoma sp., Pithomyces sp., Trichoderma sp.1, Trichoderma sp.2, Trichoderma sp.3, Rhizopus stolonifer and Stachybotris sp. were isolated by serial dilution and segment plate method. A. caespitosus, A. nidulans, Chaetomium sp., Memnoniella sp., Penicillium sp.2, Stachybotrys sp., Torula sp. were also isolated by Serial dilution method. While, A. flavus, Chaetomium sp., Memnoniella sp. and Stachybotrys sp. were isolated by Blotter paper method. Alternaria sp.2, Beltrania rhombica and Humicola sp. were not identified from Leaf Litter Fungi of Sakoli Forest by any of three methods (Table 1). During the isolation of fungi it is found that Fusarium oxysporum, A. niger, A. flavus, A. caespitosus, Penicillium sp.2, A. nidulans, Helminthosporium sp. and Monodictys fluctuata were dominant and Fusarium oxysporum was found highest colony forming fungi followed by A. niger, A. flavus, A. caespitosus, Penicillium sp.2, A. nidulans, Helminthosporium sp. and Monodictys fluctuata in Sakoli forest area. Whereas others showed least CFU (Table 2). The collected specimens are shown in fig. 4 and description recorded is as follows.

Alternaria sp. Nees. Colonies effuse, gray, dark, blackish brown or thick brown; mycelium immersed or partly superficial, hyphae pale brown, conidiophores simple, irregularly or loosely branched, brown, solitary or in fascicles; conidia caternate or solitary, dry, typically ovoid or obclavate, often with beak, pale or mid olivaceous brown, smooth or verrucose, muriform, with transverse and frequently with oblique or longitudinal septa.

Aspergillus caespitosus Raper \& Thom. Colonies growing slowly, mycelium largely submerged and extremely tough, tearing with difficulty, producing numerous dark green, hemispherical to loosely columnar heads in central colony areas, characterized particularly by clusters of irregular ovoid to elliptical and thick walled hulle cells, at first colourless, becoming reddish purplish in age, scattered unevenly or arranged in irregular concentric zones; reverse colourless changing to dark reddish purple with age, particularly beneath hulle masses; odour absent; conidial heads dark yellow green to green, generally radiate to loosely columnar, mostly $75-125 \mathrm{~m}$ in diam; conidiphores straight or slightly sinuous, mostly 250-325 $\mathrm{m}$ in length, 5-6.5 $\mathrm{m}$ in diam, thick walled, smooth when young, roughened in age, tan to brown in colour, septate just below the vesicle; vesicle slightly elongate, the upper hemisphere loosely covered by phialides, lower half sterile and often cloured, $15-20 \mathrm{~m}$ in diam; phialides beseriate; metulae 6.5-8.5 × 3.5-5 m; phialides $6.5-8 \times 3-4.5 \mathrm{~m}$, rarely larger; hulle cells abundant, thick walled, irregular; globose, spinulose, green, mostly 3.5-4.5 m, rarely larger.

Aspergillus flavus Link. Colonies growing rapidly; conidial heads yellow when young, becoming dark yellowgreen in age, in older cultures deep grey-green, reverse colourless to pale yellow brown; exudates inconspicuous, except in heavily sclerotial strains, red brown; conidial heads radiate, splitting into poorly defined columns, rarely exceeding 500-600 m, with big conidial heads yellow in the centre, greenish towards periphery; conidiophores arising separately from the substratum, 0.5-1.5 mm long, with coarsely roughened, heavy walls, colourless, broadening gradually to vesicles, $10-65 \mathrm{~m}$ in diam; metulae present predominantly, sometimes absent, only phialides (uniseriate or biseriate) arising on metulae or both types present sometimes in the same head, conidia globose to subglobose, conspicuously echinulate, yellowish green, 4.5-5.5 × 3.5-4.5 $\mathrm{m}$, sometimes elliptical when young infrequently remain so, 3.5-4.5 $\mathrm{m}$, up to $6 \mathrm{~m}$ also.

Aspergillus fumigatus Fresen. Colonies spreading dull blue-green, velvety to floccose; rapidly, white at first becoming reverse colourless to varying in shades; conidial heads columnar, compact, often densely crowded, up to $400 \times 50 \mathrm{~m}$; conidiophores short, smooth, light green, up to $300 \mathrm{~m}$ in length and $5-8 \mathrm{~m}$ in breath, septate, gradually enlarging into a flask shaped vesicle; vesicle bearing a single series of phialides; phialides closely packed, 6-8 $\times 2-3 \mathrm{~m}$; conidia globose to subglobose, green in mass, echinulate, sclerotia and cleistothecia absent.

Aspergillus nidulans Fennell \& Raper. Colonies growing well at room temperature, dark cress green in some strains from abundant conidial heads, margins thin irregular; reverse purplish red to very dark in age; exudates lacking; conidial heads slightly larger than in typical representatives of the species; cleistothecia commonly 400 $\mathrm{m}$ in diam, occasionally up to $450-500 \mathrm{~m}$; hulle cells thick walled, globose to subglobose, 1-25 m; asci subglobose to ovate, 8 spored, ascospores lenticular, red orange in colour, with two prominent pleated equatorial crests about $1 \mathrm{~m}$ wide and with convex surface conspicuously echinulate rather than smooth, 3.6-4.6×3.4-4.1 m.

Aspergillus niger Tiegh. Colonies with abundant mycelium, conidial heads carbon black or sometimes deep brownish black; reverse colourless to pale yellow; exudates limited or lacking with minute droplets; conidial heads large and black, at first globose then radiate or splitting in well defined columns in age, up to 700-800 $\mathrm{m}$ in diam; conidiophores arising directly from the substratum, smooth, non septate, thick walled, 1-2 $\mathrm{mm} \times 15-20 \mathrm{~m}$; vesicles globose, walls thick, commonly 45-75 $\mathrm{m}$ in diam, occasionally longer, beraring two series of fully packed phialides, brownish; metulae, rarely septate; phialides 7$10 \times 3-3.5 \mathrm{~m}$; conidia globose, spinulose with colouring substance, black, 4-5 m; globose to subglobose sclerotia produced in some strains, at first cream to buff, later vinaceous buff in age.

Aspergillus ochraceus Wilh. Colonies growing moderately, plane with a tough basal mycelium producing crowded conidial structures, light ochraceous buff to warm buff colour; reverse yellowish to greenish brown or reddish purple; exudates amber coloured in small droplets; conidial heads globose when young, typically adhering into two or three divergent compact columns in age, up to $750 \mathrm{~m}$ diam; conidiophores light yellow to light brown 0.8-1.5 $\mathrm{mm} \times 10-15 \mathrm{~m}$, walls thick; vesicles globose, thin walled, colourless, phialides biseriate; metulae varying in size greatly, phialides 7.5-10.5 × 2-3.7 $\mathrm{m}$; conidia globose or subglobose, delicately roughened or in some cases 
appearing smooth, 2.3-3.37 m; sclerotia abundant, globose to subglobose, white to light pink when young, later vinaceous purplish, up to $1 \mathrm{~mm}$ in diam.

Aureobasidium sp. Viala \& Boyer. Colonies effuse, white or creamy, later becoming black at least in part and usually slimy; mycelium mostly immersed, variable in thickness; conidiophores branched, flexuous, at first hyaline, becoming mid to dark brown, smooth, thick walled; conidia aggregated in slimy mass, semiendogenous, pleurogenous, simple, ellipsoidal or ovoid, colourless, smooth, aseptate, each completely encased in slimy coat; secondary conidia produced by yeast like budding of primary conidia; dark hyphal portions many act as chlamydospores or fragment like arthroconidia.

Beltrania rhombica Penzig. Colonies brown to black; mycelium immersed or superficial; Setae smooth, usually less than $200 \mathrm{~m}$ long, occasionally longer, 4-6 m near the base; conidiophores upto $130 \mathrm{~m}$ long, 4-8 $\mathrm{m}$ wide; separating cells ellipsoidal or obovoid, pale, 6-15 × 3-6 $\mathrm{m}$; conidiogenous cells integrated, terminal, polyblastic, denticulate; conidia spicate, biconic, symmetrical, proximal end V-shaped, 15-30 × 7-14 m (without appendages); appendages 3-20 m long, $2 \mathrm{~m}$ wide at the base, tapering to a pointed tip.

Chaetomium sp. Kunze \& Schmidth. Mycelium thread like, thick or thin walled, septate, hyaline to yellowishbrown, septate; perithecia superficial, attached to the substrate by rhizoids, ostiolate, translucent to opaque to dark coloured, oval, subglobose, barrel shaped, clothed with dark setaeform or hair like appendages; hairs terminal and lateral, myceloid, stiff or coiled, branched or unbranched, smooth or ornamented; asci cylindrical, clavate to club shaped, 4-8 spored, unitunicate, hyaline, evanescent; paraphyses greatly reduced; ascospores unicellular, olive-brown to chocolate-brown, globose to subglobose to broadly lemon shaped, with a single germ pore, rounded to umbonate or faintly apiculate at one or both ends, column like mass of black ascospores liberating from ostiole; rarely producing aleurospores or chlyamydospores.

Curvularia sp. Kunze \& Schmidt. Colonies effuse, brown, grey or black, hairy, cottony or velvety; mycelium immersed in natural substrata; erect, black, cylindrical, sometimes branched, conidiophores indeterminate, continuing growth sympodially, straight or flexuous, often geniculate, sometimes later becoming intercalary, sympodial, cylindrical or occasionally swollen, cicatrized; conidia porospores, solitary, acropleurogenous, simple, often curved, clavate, ellipsoidal, broadly fusiform, obovoid or pyriform with 3 or more transverse septa, pale or dark brown, often with some cells (usually one end ones) paler than the other cells, sometimes with dark bands at the septa, smooth or verruculose; hilum distinctly protuberant in some species of scarcely or not at all protuberant in others.

Fusarium oxysporum Schlecht. Mycelium white or peach, but usually with a purple or violate tinge; reverse colourless to dark purple; conidiophores unbranched or sparsely branched, monophialidic; microconidia usually abundant, mostly 0 -septate, oval ellipsoidal, kidney shaped or straight, produced on simple lateral phialides, solitary on free conidiophores never form in chains, 5-12 $\times 2.5-3.5$ $\mathrm{m}$; macroconidia 2-5 septate, spindle to fusiform, curved or almost straight, pointed at both the ends, definite or weakly pedicellate, $27-60 \times 3-5 \mathrm{~m}$; chlamydospores mostly terminal, globose smooth or roughened.

Fusarium sp. Link. Mycelium extensive and cottony in culture, often with some tinge of pink, purple or yellow, in the mycelium or medium; conidiophores variable, slender and simple, or stout, short, branched irregularly or bearing a whorl of phialides, single or grouped into sporodochia; conidia (phialospores) hyaline, variable, principally of two kinds, often held in small moist heads; macroconidia several-celled slightly curved or bent at the pointed ends, microconidia 1-celled, oblong or slightly curved; parasitic on higher plants or saprophytic on decaying plant material.

Helminthosporium sp. Link ex Fr. Mycelium dark, often in substrate; conidiophores single or clustered, tall, erect, brown, simple; conidia (porospores) develop laterally through pores beneath septa while apex of conidiophores is still growing whorls, single, subhyaline to brown, obclavate, phragmosporous, pseudoseptate, with prominent basal scar; parasitic or saprophytic.

Humicola sp. Traaen. Colonies effuse, cottony, sometimes, funiculose, at first white, later grey, brown with age; mycelium superficial and immersed; intercalary chlamydospores sometimes present; conidiophores microor semi-macronematous, unbranched or irregularly branched, straight or flexuous, colourless to pale golden brown, smooth; conidia (aleuroconidia) solitary, dry, acrogenous, simple, typically spherical, occasionally obovoid or pyriform, pale to mid golden brown, 1-celled smooth; phialidic conidia may be catenate, small, colourless, smooth 1-celled.

Memnoniella sp. Hohnel. Colonies effuse, black, velvety or powdery; mycelium partly superficial or immersed; conidiophores macronematous, mononematous, unbranched swollen at the apex, pale to mid grey, olivaceous or brown, smooth or minutely verruculose, often covered with dark granules; conidia acrogenous, catenate, spherical, hemispherical, grey dark brown or black, slightly flattened in one plane, smooth or verrucose.

Monodictys fluctuata S. Hughes. Colonies becoming dark grey with age; mycelium thin, hyaline and thick; conidiophores both terminal and lateral, light brown, 5.2-8 $\mathrm{m}$ wide, flattened end bearing conidia of very variable shape and size; conidia dark brown, 2-14 celled, often constricted at the septa, at first smooth, later verruculose, roughly subspherical with straight or oblique septa, the size of the different types of conidia varies, up to $40 \mathrm{~m}$ in diameter.

Penicillium adametzi Zaleski. Colonies growing 30-35 mm diameter on MEA in 7 days at $25^{\circ} \mathrm{C}$, plane very deep, funiculose; mycelium white to cream coloured; conidiophores mostly arising from funicles, sometimes from aerial or surface hyphae; stipe short, smooth, monoverticillate, non-vesiculate or slightly swollen apices; phialides usually in compact verticils of 5-8, ampulliform, 6-8 × 2-2.2 m; conidia spheroidal, 1.8-2.5 m diam, smooth to finely roughened, borne in short, poorly defined columns. 


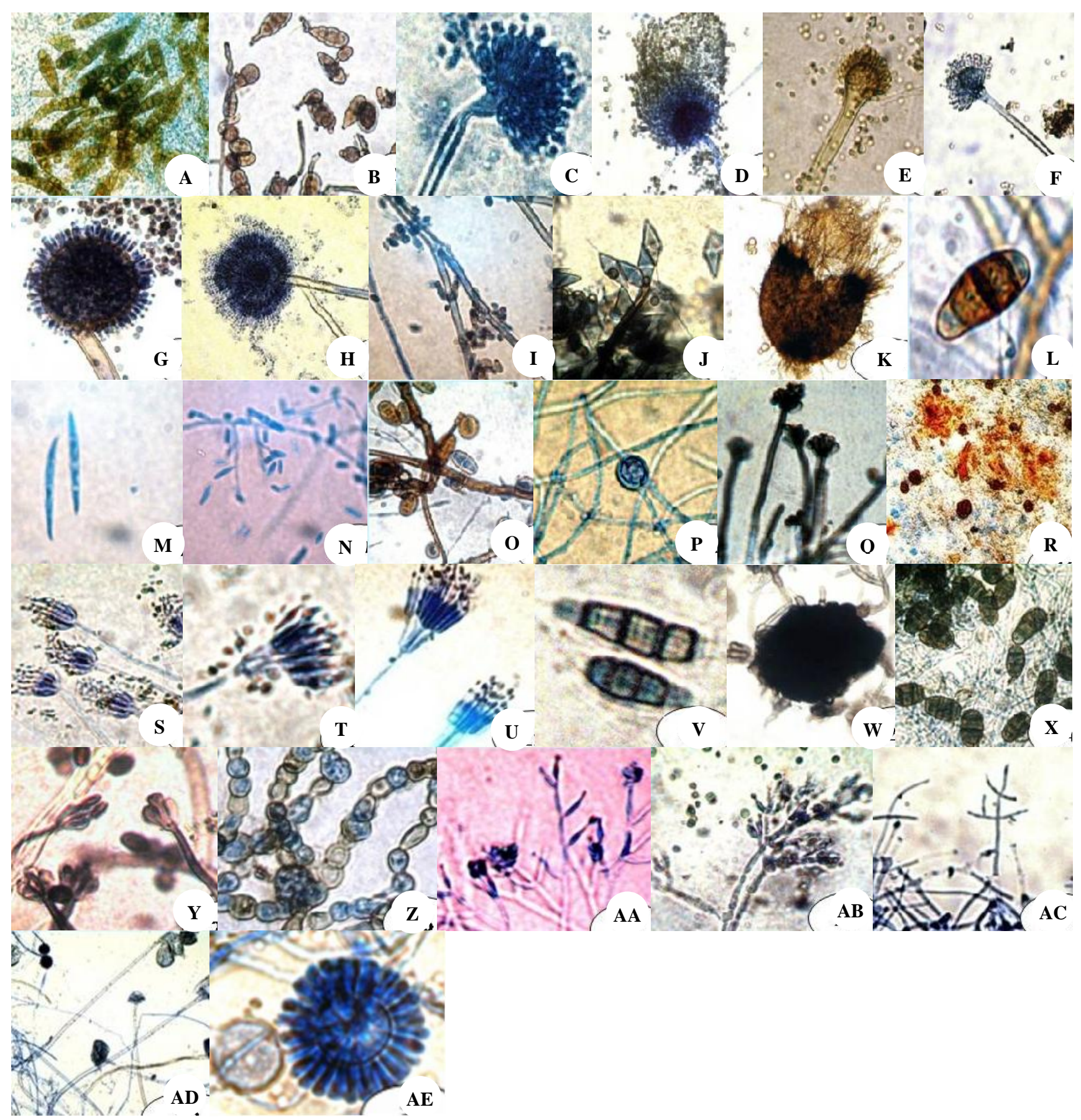

Figure 4. Schematic diagram of isolated fungi. A. Alternaria sp.1, B. Alternaria sp.2, C. Aspergillus caespitosus, D. Aspergillus flavus, E. Aspergillus fumigatus, F. Aspergillus nidulans, G. Aspergillus niger, H. Aspergillus ochraceus, I. Aureobasidium sp., J. Beltrania rhombica, K. Chaetonium sp., L. Curvularia sp., M. Fusarium oxysporum, N. Fusarium sp.1, O. Helminthosporium sp., P. Humicola sp., Q. Memnoniella sp., R. Monodictys fluctuata, S. Penicillium adametzi, T. Penicillium sp.1, U. Penicillium sp.2, V. Pestalotia sp., W. Phoma sp., X. Pithomyces sp., Y. Stachybotrys sp., Z. Torula sp., AA. Trichoderma sp.1, AB. Trichoderma sp.2, AC. Trichoderma sp.3., AD. Rhizopus stolonifer, AE. Syncephalastrum sp.

Penicillium sp. Link. Colonies variously coloured, usually blue-green, texture variable, zonate or azonate; vegetative hyphae creeping, septate; exudates present or absent; reverse uncoloured or variously coloured; conidiophores usually conspicuous, more or less erect, sometimes aggregated into synnemata, hyaline, rough or smooth, septate, sometimes branching at or near apex, branches divergent or adpressed to the main conidiophores axis, series of branches giving characteristics brush-like penicullus; phialides borne in groups directly at the apex of the conidiophores or branches of the conidiophores, ampulliform or acerose, hyaline; conidia borne in long 
chains, globose to ovoid, sometimes bacillar, hyaline to darkly pigmented, smooth or roughened; sclerotia produced in some species.

Pestalotia sp. Steyaert. Colonies compact or effuse, buff, grayish brown, blackish brown or black; mycelium immersed, branched, septate, hyaline to pale brown; conidiophores hyaline, branched and septate at the base and above, cylindrical or lagerniform, formed from the upper cells of the pseudoparenchyma; conidia fusiform, straight or slightly curved, 4-euseptate, base simple or rarely with branched appendage, apical cell conic, hyaline, with 2 or more apical, simple or branched, spathulate or espathulate appendages, median cells brown, sometimes versicoloured, thick walled, smooth or verruculose.

Phoma sp. Sacc. Colonies variable in form and growth; aerial mycelium white, grey-green, olivaceous or black often sectoring, zonate or azonate; mycelium immersed, branched septate hyaline or pale brown; reverse buff, yellow, saffron, reddish, greenish brown, dark brown; pycnidia unilocular, rarely multilocular, brown, globose, separate or aggregated, occasionally confluent mostly thin, 1-3 celled thick, pale to medium brown; ostioles single or occasionally confluent and multi-ostiolate, central, not papillate; conidiophores present few species only, then either filiform, septate and branched or short, irregularly branched and ramified respectively; conidia slimy, hyaline, aseptate or occasionally one septate, thin walled, often guttulate, ellipsoid, cylindrical, fusiform, pyriform or globose, smooth.

Pithomyces sp. Berkeley \& Broome. Colonies punctiform or effuse, yellow, olive green, brown or black; mycelium all or mostly superficial; conidiophores branched, straight or flexuous, subhyaline, pale olive to brown, smooth or verruculose; conidia solitary, dry, simple, ellipsoidal, clavate, limoniform, obovoid, oblong, rouded at ends, pyriform, detached through fracture of the denticle, a part or which often remains attached to the conidium of short pegs, straw coloured to blackish brown, smooth, echinulate or verruculose, up to 13-transversely septate, often with one or more oblique or longitudinal septa.

Stachybotrys sp. Corda. Colonies effuse, usually black or blackish green; mycelium superficial, immersed; hyphae sometimes forming ropes; conidiophores macronematous, unbranched or cymosely branched, each stipe and branch straight or flexuous, colourless greey brown, olivaceous brown or black, smooth or verrucose; phialides forming in clustures and in succession, ellipsoidal or subclavate, hyaline or pigmented, determinate, usually with very small opening and no collarette; conidia aggregating in large, slimy often black and glistening heads, acrogenous, simple, cylindrical or oblong, rounded at one end or both ends, ellipsoidal, reniform or subsperical, gray, greenish, dark brown \pm opaque, smooth or verrucose, covered with dark granules or longitudinal striations, or none, 1-celled, released in basipetal succession, new conidia arise after the release of mature conidia from phialide neck.

Torula sp. Peersoon ex Fries. Colonies effuse, sometimes small and discrete, olive, brown, dark, blackish brown or black, often velvety; mycelium superficial and immersed; micronematous or semi-macronematous, unbranched or irregularly branched, straight or flexus, subhyaline to mid browm, smooth or verruculose; conidia (phragmoconidia) blastic, dry, in simple or branched chains arising from surface of upper half of the conidiogenous cells, cylindrical with rounded ends, ellipsoidal, subspherical, cylindrical or fusiform, brown or olivaceous brown, smooth, verruculose or echinulate, with 0 -several transverse septa, breaking into phragmo-conidia.

Trichoderma sp. Pers. Colonies growing rapidly or variable depending on species, turning from watery translucent smooth surface to floccose to compactly tufted pustules; Conidiophores hyaline, much branched, not verticillate; phialides single or in groups; conodia (philospores) hyaline, 1-celled, ovoid, borne in small terminal clusters; usually easily recognized by its rapid growth and green patches or cushions of conidia; saprophytic in soil or on wood.

Rhizopus stolonifer Ehrenberg. Colonies white at first, turning brownish black, stolons spreading, internodes brown, with well branched brown rhizoids at each node; sporangiophores in clusters of 3-10, unbranched, 0.4-4 mm long and 24-42 m, white, becoming pale to dark brown at maturity; sporangia globose, hemispherical, granular, olivaceous, black, 100-300 m, columella hemispherical, very often becoming pilate, $45-100 \mathrm{~m}$ or bigger; sporangiospores irregular, round to oval, angular, straight, grey, striate, $9-12 \times 7.5-8.1 \mathrm{~m}$; zygospores round to oval, 160-220 m, exine brown-black, verrucose; clamydospores absent.

Syncephalastrum sp. Schroet. Colonies fast growing, light to dark grey; mycelium wide spreading coenocytic, profusely branched; sporangiophores erect, lacking rhizoids or often produce adventitious rhizoides, branching irregular or \pm racemosely branched, often curved with branches, each branch apically dilated to form globose vesicle; vesicle globose to ovoid, separating from sporangiophores by septum, bear merosporangia directly over their entire surface; merosporangia rod shaped, many spored without a basal cell, wall evanescent at maturity; sporangiospores uniseriately arranged, globose or ovoid, formed by simultaneous cell delimitation; zygospores globose, dark brown, rough with broad shallow pointed projections, formed in the aerial mycelium between nearly equal suspensors.

\section{Discussion}

Present investigation carried out for the isolation and identification of forest leaf litter fungi to study fungal diversity of Bampewada and Sakoli forest area in Bhandara district of Maharashtra state. Studies of fungal diversity of Bampewada and Sakoli forest area in Bhandara district have not been carried out until. Although, the fungal genus found in present study is very common in forest habitat throughout the world (Paulus et al. 2006; Turkoglu et al. 2007). Many fungal species were found in litters in different forest types (Yao et al. 1997). Fifty-seven taxa comprising of 18 ascomycetes fungi were identified with direct identification method in rain forest (Parungao et al. 2002). Seventy fungi were reported in the temperate forest 
of Japan (Osono et al. 2002). Similarly many workers isolated number of fungi from different decomposing matter. Warcup (1957) isolated Fusarium sp., Penicillium sp. and Mucor sp. during decay when soluble components of straw are available. Elkady et al (1981) isolated $37 \mathrm{sp}$. of mesophilic fungi belonging to 19 genera from 15 wheat straw samples by using dilution plate technique. Kiran, Usha and Garecha (1982) isolated Helmithosporium sp. and Fusarium oxysporum from mushroom compost. Likewise Fusarium sp., Mucor sp., Penicillium sp. and Trichoderma sp. were isolated by Harper and Lynch (1984).

In this study, total 31 fungi were found in two forests namely Bampewada and Sakoli. The results suggested that the diversity of fungi associated with forest leaf litter were varied with types and climate of the forests. Number of fungal species from Sakoli forest was more than that from the Bampewada forest (Table 2). The reason may be that the quality of litters, different microenvironments and other characteristics in the Sakoli forest provided more resources for fungi than in Bampewada forest. For the significant isolation of fungi more than one method should be applied (Ramesh and Chalannavar 1998). These conclusions also correlate with the present study. The isolated fungi have shown variability in their occurrence and number. During the study it was found that Fusarium oxysporum and Trichoderma sp.1 were more common in segment plate method. From this observation it is concluded that the abundance of fungal species are slightly different in the selected areas. The total number of fungal colonies observed and identified was more in the leaf litter of Sakoli forest. This phenomenon could be even found in different parts of a plant (i.e. leaves, leaf midribs and petioles) (Hyde et al. 2000). This indicated that some fungi may preferentially develop in certain tissue types (Huang et al. 1998).

The decomposition of plants began with the intrusion of the still growing frond by the fungal pathogens (Osono et al. 2002). After falling down, the leaf litter was then decomposed by a series of fungi. The investigation indicated that the fungi diversity in the same forest litter layer in Sakoli forest was notably higher than that in Bampewada forest, suggesting that fungi diversity was related with litter quality. The reason may be that the diverse litter quality between two types of forest communities decreased with the decomposing process (Tian et al. 2002), leading to fungi richness. Litter decomposition included a processed, the physical and biological complexity of litters generally increased, leading to an increase of decomposer diversity (Huang et al. 1995; Nazim et al. 2013). Most decomposers were of litter specific ( $\mathrm{Li}$ et al. 2000). This may be due to the fact that the fungi have their own ecological characteristics to litters. These results were very similar to previous work carried out by many workers on isolation of leaf litter fungi and conclude that members of Zygomycetes and Ascomycetes play active role in the decomposition process. Borkar (2014) observed that most of the fungi isolated from degrading biomass are the members of ascomycetes where as very few fungi belongs to other groups like Zygomycetes and Basidiomycetes. Also, Mehrotra and
Aneja (1979) reported similar observations when they isolated mycoflora of Chenopodium leaf litter. The highest number of fungal species could be detected by serial dilution method compared to other two methods which emphasize the importance of using a combination of several cultural methods for studying fungal diversity of decaying plant substrates.

In conclusion, the results of the study suggest that the diversity of fungi in Sakoli forest is higher than the Bampewada forest. All total 31 species belonging to 20 genera of leaf litter fungi were found in selected forest area i.e. Sakoli forest and Bampewada forest. Overall, greater diversity of fungi was observed in forest area and varied between the methods employed. Based on results, sampling design to capture the diversity of microfungi in Sakoli forest area should include litter of many different tree species or leaf types as well as samples should collect from different sites. For a single time point sample, many more species of microfungi were recovered from leaf litter using serial dilution method and segment plate method rather than Blotter paper method. This further emphasizes the need for assessment of fungal communities using a molecular and biochemical techniques.

\section{ACKNOWLEDGEMENTS}

The authors are thankful to Head of Department of Botany and the authorities of R.T.M. Nagpur University, Nagpur for providing necessary facilities throughout the course of this investigation.

\section{REFERENCES}

Ainsworth GC, Sparrow FK, Sussan AS. 1972. The Fungi, an Advanced Treatise. Vol. IV A. Academic Press, New York.

Barnett HL, Hunter B. 1972. Illustrated Genera of Imperfect Fungi. $3^{\text {ed }}$. Burgess Pub. Co., Minneapolis.

Borkar KM, Thakre RP. 2014. Effect of temperature, $\mathrm{pH}$ and substrates on CMCase enzyme activity of thermophilic fungus Humicola insolens. Intl J Life Sci A2: 91-94.

Change ST, Miles PG. 2004. Mushrooms cultivation, national value, medicinal effect and environmental impact. 2nd ed. CRC Press, New York

Elkady IA, Abdel-Hafez SII, Moubasher MH. 1981. Survey on the cellulose decomposing fungi of wheat straw in Egypt. Mycopathologia 76: 59-64.

Fulzele AA, Thakre RP. 2002. The cellulolytic activity of some biodegrading fungi. Biotechnology of Microbes and Sustainable Utilization. Vedams eBooks (P) Ltd., New Delhi.

Gates GM, Ratkowsky DA, Grove SJ. 2005. A Comparision of macrofungi in young Silvicultural regeneration and mature forest at the Warra LTER siet in the southern forests of Tasmania. Tasforests 16: 127.

Grigal DF, McColl JG. 1977. Litter decomposition following forest fire in northeastern Minnesota. J Appl Ecol 14: 531-538.

Harper SHT, Lynch JM. 1984. Effects of microbial colonization of barley (Hordeum vulgare L.) roots on seedling growth. J Appl Bacteriol 57: 131-137.

Huang J, Chen L, Han X. 1998. Change of organic matter in the decomposing oak twigs in the temperate forest eco-systems. Acta Botanica Sinica 40: 362-369.

Huang J, Han X. 1995. A review on the biogeochemical cycling in forest ecosystems: Theories and methods. Chinese Bull Bot 12: 145-223.

Hughes SJ. 1989. New Zealand fungi 33, some new species and new record of dematiaceous hyphomycetes. N Z J Bot 27: 449-459. 
Hutchinso HB, Richards EH. 1921. Artificial farmyard manure. J Ministry Agri 28: 398

Hyde KD, Alias SA. 2000. Biodiversity and distribution of fung associated with decomposing Nypa fruticans. Biodivers Conserv 9 393-402.

Isidorov V, Jdanova M. 2002. Volatile organic compounds from leaves litter. Chemosphere 48: 975-979.

Li BO, Yang C, Lin P. 2000. Ecology. Higher Education Press, Beijing.

Manoharachary C, Sridhar K, Singh R, Adholeya, Suryanarayanan TS, Rawat S, Johri BN. 2005. Fungal biodiversity: Distribution, conservation and prospecting of fungi from India. Curr Sci 89: 58-71.

Mehrotra RS, Aneja KR. 1979. Microbial decomposition of Chenopodium album litter. Succession of decomposers. J Indian Bot Soc 58: 189195.

Mueller, G. M. Bills, G. F.Foster, M. S. (eds) 2004. Biodiversity of fungi: inventory and monitoring methods. Elsevier, Amsterdam.

Mueller, G. M., Schmit, J.P. 2007. Fungal biodiversity: what do we know? What can we predict? Biodivers Conserv 16 (1): 1-5.

Nazim K, Ahmed M, Shaukat SS, Khan MU. 2013. Seasonal variation of litter accumulation and putrefaction with reference to decomposers in a mangrove forest in Karachi, Pakistan. Turk J Bot 37: 735-743.

Osono TS, Hobara S, Fujiwara K, et al. 2002. Abundance, diversity, and species composition of fungal communities in a temperate forest affected by excreta of the Great Cormorant Phalacrocorax carbo. Soil Biol Biochem 34: 1537-1547.

Parungao MM, Fryar SC, Hyde KD. 2002. Diversity of fungi on rainfores litter in North Queensland, Australia. Biodivers Conserv 11: 11851194.

Paulus B, Kanowski J, Gadek P, Hyde KD. 2006. Diversity and distribution of saprobic microfungi in leaf litter of an Australian tropical rainforest. Mycol Res 110 (12): 1441-1454.

Ramesh CH, Chalannavar RK. 1998. Preliminary studies on decomposition of leaf litter of Citrus aurantium: comparative evaluation of different isolation techniques. Indian Phytopathol 51 (4): 385-386.
Raper KB, Thom C. 1949. A Manual of Penicillia. Williams and Wilkins Company, Baltimore.

Rawling TE. 1933. Phytopathological and Botanical Research Methods. John Wiley and Sons, London.

Saksena SB. 1955. Ecological factors governing the distribution of microfungi in forest soil of Sagar. J Indian Bot Soc 34: 262-298.

Saravanan R, Sivakumar T. 2013. Biodiversity and Biodegradation potentials of Fungi isolated from Marine systems of East Coast of Tamil Nadu, India

Int J Curr Microbiol App Sci 2 (7): 192-201.

Senthilarasu G. 2014. Diversity of agarics (gilled mushrooms) of Maharashtra, India. Curr Res Environ Appl Mycol 4 (1): 5878

Sutton BC. 1980. The Coelomycetes, fungi imperfectii with pycnidia, ascervulii and stromata. CMI, Kew, England.

Tian X, Tateishi T. 2002. Relative roles of microorganisms and soil animals on needle litter decomposition in a subalpine coniferous forest. Acta Phytoecology Sinica 26: 257-263.

Tokumasu S, Tubaki K, Manoch L. 1997. Microfungal communities on decaying pine needles in Thailand. In: Janardhanan KK, Natarajan KR, Hawksworth DL. (eds.) Tropical Mycology. Science Publishers Inc, Enfield, New Hampshire.

Turkoglu A, Kanlik A, Gezer K. 2007. Macrofungi of Çameli District (Denizli-Turkey). Turk J Bot 31: 551-557.

Van der Walt JP, Yarrow D. 1984. Methods for the isolation, maintenance, classification and identification of yeast. In Kreger-van Rij NSJ (ed.). The Yeast: A Taxonomic Study. 3rd ed. Elsevier, Amsterdam.

Von Arx JA. 1981. The genera of fungi sporulating in pure culture. 3rd ed. J Cramer, Vaduz, Germany.

Warcup JH. 1957. Studies on the occurrence and activity of fungi in a wheat-field soil. Trans Br Mycol Soc 40: 237-262.

Yao T, Yang J. 1997. Microbial Ecology of litter and Soil in Forest. J Northwest For Coll 12 (4): 97-103. [Chinese] 